\title{
Role of microbubbles coupling fibrous-bed bioreactor in butyric acid production by Clostridium tyrobutyricum using Brewer's spent grain as feedstock
}

Jin Huang ( $\nabla$ huangjin979@zjut.edu.cn )

Zhejiang University of Technology https://orcid.org/0000-0002-9356-0589

Linqi Zhao

Zhejiang University of Technology Chaohui Campus: Zhejiang University of Technology

Gaoya Sun

Zhejiang University of Technology Chaohui Campus: Zhejiang University of Technology Jing Jiang

Zhejiang University of Technology Chaohui Campus: Zhejiang University of Technology

Li Chen

Zhejiang University of Technology Chaohui Campus: Zhejiang University of Technology

\section{Research}

Keywords: Butyric acid, Clostridium tyrobutyricum, microbubbles, ATPase, Brewer's spent grain

Posted Date: January 28th, 2021

DOI: https://doi.org/10.21203/rs.3.rs-152354/v1

License: (a) (i) This work is licensed under a Creative Commons Attribution 4.0 International License.

Read Full License 


\section{Abstract}

Background: Microbubbles coupling fermentation is an efficient technology for improving oxygen transfer to aerobic microorganisms in a bioreactor containing an air sparger. However, the effect of this technology on anaerobic Clostridium tyrobutyricum, whose morphology develops viscous broth rheology and lumps impeding the nutrient mass transfer, has not been reported yet. Therefore, in this study, we evaluated the role of microbubbles coupling fibrous-bed bioreactor (MBFBB) on butyrate production by immobilized Clostridium tyrobutyricum cells obtained from renewable feedstock brewer's spent grain (BSG).

Results: Compared with the conventional FBB fermentation, two-fold shorter fermentation time and twofold higher butyrate productivity were achieved by MBFBB-immobilized $C$. tyrobutyricum cells. Furthermore, long-term stability and reliability for butyrate production were obtained in ten cycle process using BSG hydrolysate. Finally, fed-batch fermentation using BSG hydrolysate produced a high butyrate titer of $43.68 \mathrm{~g} / \mathrm{L}$, with a significantly higher selectivity of 11.67 , reducing the production cost of downstream processing. The results indicated that butyrate productivity of 4.21 and $4.36 \mathrm{~g} / \mathrm{L} \cdot \mathrm{h}$ were successfully obtained in BSG and glucose medium, thereby representing the highest productivity reported to date in $C$. tyrobutyricum. To our knowledge, this is also the first report related to microbial production of butyrate from brewer's spent grain.

Conclusion: The MBFBB-based fermentation process with BSG is a robust and ecofriendly technique, which would provide insights into future development of commercial biobutyrate production for the animal feed supplement market.

\section{Highlights}

1. Production of butyrate from brewer's spent grain,an abundant industrial waste biomass

2. tyrobutyricum in MBFBB was used for butyrate fermentation

3. Cells in MBFBB yielded a higher ATPase activity, sugar utilization rate and productivity

4. The fermentation process from BSG hydrolysate has long-term stability and reliability

5. The MBFBB-based process was economic and attractive for waste biomass biorefinery

\section{Background}

Butyric acid, an oligomerize fatty acid, has been widely adopted in the fields of food, animal feed supplement, pharmaceutical, perfume, and industrial chemicals, with a global market of more than $8 \times 10^{4}$ metric tons per year [1]. However, the industrial production of butyrate is currently dominated by the chemical oxidation of petroleum-based butyraldehyde, which caused environmental pollutions and is also not favored for the food and feed industries [2,3]. In contrast, naturally derived butyrate through microbial fermentation has emerged as a promising alternative and extensively investigated for ecofriendly characteristics. Clostridial species are the most feasible strains for industrial butyrate bio- 
production, especially, Clostridium tyrobutyricum due to its higher selectivity and tolerance to a high concentration of products [4-6]. Besides, the conventional fermentation technology for industrial production of butyric acid is not economically feasible due to cytotoxicity and byproducts generation, resulting in a low titer, yield, and productivity of butyrate [7]. By using an immobilized-cell bioreactor, the product inhibition could be partially relieved by in situ product removal and cell immobilization. However, the butyrate productivity in typical immobilized-cell fermentation process significantly decreases over extended operation periods, resulting from dead cell lumps accumulation and poor mass transfer under high cell density.

Microbubbles coupling fermentation is an efficient technology making breakthrough progress in recent years and has been widely adopted for the production of bioethanol [8], cellulase [9], and polyhydroxy butyrate [10]. Microbubbles exert outstanding characteristics than normal bubbles. The microbubbles dispersion generator can reduce the size of normal sparged gas bubbles from 3-5 $\mathrm{mm}$ to approximately 20-100 $\mu \mathrm{m}$, leading to lower rising velocity than normal bubbles [11,12]. Additionally, the negatively charged surface prevents the microbubbles from coalescence, allowing them to persist in water for a longer period of two weeks [13]. Further, the microbubbles possess high internal pressure and a large specific surface area, which can significantly improve the mass transfer rate from bubbles into the aqueous phase resulting in higher dissolved gas concentration. Subsequently, the microbubbles can prolong the reactivity, increase the dissolved nutrient concentration, and the synthetic efficiency of target metabolites. It has been reported that microbubbles sparged fermenters could be energy efficient up to $0.01 \mathrm{~kW}$ per $\mathrm{m}^{3}$ of fermentation capacity, lowering overall operating costs [14].

In contrast, the replacement of pure carbon source sugar with low-cost waste biomass (e.g., corn fiber, waste paper, oilseed rape straw, and wheat straw) could notably reduce the butyrate bio-production cost [15-18]. Although various waste biomasses have been explored to produce butyrate, the complexity of lignocellulosic biomass, especially lignin and the crystalline structure of cellulose, requires an additional process, such as pretreatment and hydrolysis to remove biomass recalcitrance forces and polymeric carbohydrate breakdown. Therefore, suitable and sustainable biomasses with high monosaccharide contents are highly necessary to simplify pretreatment and increase the titer, yield, and butyrate productivity. Unlike biomasses from the seasonal agricultural crops, Brewer's spent grain (BSG) is a lignocellulosic waste biomass available throughout the year in beer factories, with low cost and large amounts. The worldwide annual production of BSG has been estimated to be approximately $38.6 \times 10^{6}$ MT per year [19]. BSG is mainly composed of fibers with its sugar content corresponding to half of the BSG composition on a dry weight basis. Lignin and proteins also contribute to the BSG composition in significant amounts. Hence it is mostly employed by local farms for cattle feed usage. Recently, BSG has been applied as a raw material to produce value-added compounds, such as xylitol, methane, ethanol, butanol, and biohydrogen. It should be noted that there is no report on the use of BSG as a substrate for biobutyrate production to reduce product cost.

The aim of this study was to evaluate the role of microbubbles coupling fibrous-bed bioreactor (MBFBB) on butyrate production by immobilized Clostridium tyrobutyricum cells obtained from renewable 
feedstock brewer's spent grain (BSG). This technology yielded a high cellular ATPase activity and sugar utilization rate of immobilized cells and enhanced butyrate productivity. Enhanced butyrate production from BSG hydrolysate was demonstrated in repeated-batch and fed-batch processes with $C$. tyrobutyricum cells immobilized in MBFBB. Finally, the techno-economic analysis proved the feasibility and advantages of our established fermentation process for butyrate production from industrial waste biomass BSG.

\section{Results}

\section{Fermentative production of butyric acid by immobilized Clostridium tyrobutyricum in FBB or MBFBB}

The fermentative productions of butyric acid by immobilized $C$. tyrobutyricum in FBB or MBFBB were evaluated and compared, taking glucose as the carbon source (Table 1). Although the titers and yields of butyrate and acetate, as well as the selectivity, were similar in both FBB and MBFBB, the fermentation time was significantly shortened in MBFBB $(5 \mathrm{~h})$ compared to FBB (12 h). This two-fold decrease led to an increment in butyrate productivity from $2.24 \mathrm{~g} / \mathrm{L} \cdot \mathrm{h}$ to $4.36 \mathrm{~g} / \mathrm{L} \cdot \mathrm{h}$, and the glucose utilization rate was simultaneously improved from 1.25 to $2.35 \mathrm{~g} / \mathrm{L} \cdot \mathrm{h}$ for about two folds. However, the selectivity

( $\left.g_{\text {butyrate }} / g_{\text {acetate }}\right)$ in MBFBB did not vary from FBB, which might be due to the negligible butyrate inhibition and inefficient reutilization of acetate within a shorter fermentation time in MBFBB. Similarly, when BSG hydrolysate was applied as a substrate in MBFBB, the sugar utilization rate was $2.87 \mathrm{~g} / \mathrm{L} \cdot \mathrm{h}$, and the butyrate productivity was $4.21 \mathrm{~g} / \mathrm{L} \cdot \mathrm{h}$, which was the highest ever reported for butyrate fermentation [6]. Therefore, microbubbles exhibited positive effects on FBB fermentation and significantly improved the mass transfer rate between gas and liquid.

Table 1 Fermentative production of butyric acid by immobilized Clostridium tyrobutyricum in FBB or MBFBB. 


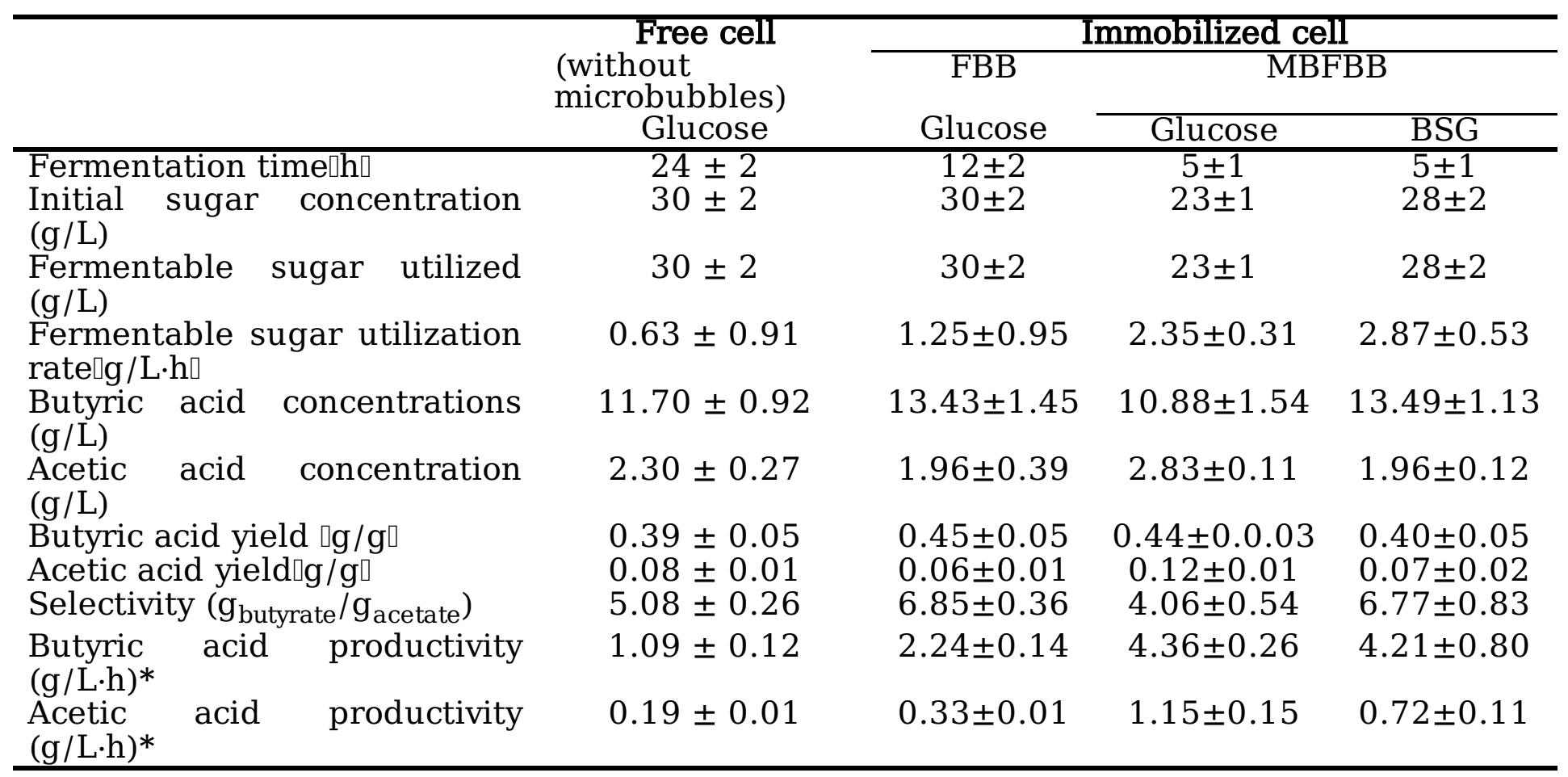

*Butyric acid and acetic acid productivity of immobilized-cell fermentation was calculated with the medium volume of $500 \mathrm{~mL}$ in the FBB, instead of the total liquid medium volume of $2 \mathrm{~L}$ in the reactor system used.

\section{Effects of microbubbles on cellular ATPase activity and sugar utilization rate of Clostridium tyrobutyricum immobilized in FBB}

The immobilized cells were harvested after $1,3,5$, and $7 \mathrm{~h}$ in repeated batch fermentations of MBFBB and FBB to evaluate the potential effect of microbubbles on Clostridium tyrobutyricum. The cellular ATPase activities were measured, and the results are illustrated in Fig. 1a. It was observed that the immobilized cells in the microbubble coupling FBB had a significantly higher ATPase activity than the nonmicrobubbles coupling FBB $(60.16 \mathrm{U} / \mathrm{g} v s .18 .2 \mathrm{U} / \mathrm{g}, 73.37 \mathrm{U} / \mathrm{g} v s .35 .54 \mathrm{U} / \mathrm{g}, 87.19 \mathrm{U} / \mathrm{g} v s .45 .76 \mathrm{U} / \mathrm{g})$, and the cellular ATPase activities were increased by $230.5 \%, 106.4 \%, 90.5 \%$ after $1,3,5 \mathrm{~h}$, respectively. When the fermentation ended at $7 \mathrm{~h}$, the cellular ATPase activities of the immobilized cells in MBFBB significantly decreased. However, the cellular ATPase activities of the immobilized cells in FBB continuously increased with the prolonged fermentation time to maintain cell growth and proton transmembrane flux balance. Besides, the immobilized cells in MBFBB had a significantly higher cellular sugar utilization rate than FBB $(0.050 \mathrm{~g} / \mathrm{g} \cdot \mathrm{h} v s .0 .043 \mathrm{~g} / \mathrm{g} \cdot \mathrm{h}, 0.067 \mathrm{~g} / \mathrm{g} \cdot \mathrm{h} v s .0 .044 \mathrm{~g} / \mathrm{g} \cdot \mathrm{h}, 0.076 \mathrm{~g} / \mathrm{g} \cdot \mathrm{h} v s$. $0.049 \mathrm{~g} / \mathrm{g} \cdot \mathrm{h}$ ), and the cellular sugar utilization rates increased by $16.2 \%, 52.3 \%$, and $55.1 \%$, respectively (Fig. 1b). With the progress of fermentation, both the cellular sugar utilization rates of the immobilized cells in microbubble coupling FBB and that in non-microbubbles coupling FBB significantly increased before the end of the fermentation.

Fig. 1 Effects of microbubbles on cellular ATPase activity (A) and sugar utilization rate (B) of Clostridium tyrobutyricum immobilized in MBFBB. Results are expressed as the mean value and standard deviation 
of duplicate samples. (p-value $*<0.05 ; * \star<0.01 ; * \star *<0.001 ;)$

\section{Repeated-batch fermentation from BSG hydrolysate in MBFBB}

The repeated-batch fermentation of BSG hydrolysate was first conducted in MBFBB to determine the long-term stability and reliability of the system. As depicted in Fig. 2a, no lag phase was found at the beginning of each cycle, which clearly indicated the high metabolic activities and adaptability of immobilized cells in the fresh medium. Most importantly, stable and reliable butyrate production was achieved for ten cycles and the titer, yield, and volumetric productivity were between 8.83 to $14.95 \mathrm{~g} / \mathrm{L}$, 0.30 to $0.48 \mathrm{~g} / \mathrm{g}$, and 2.85 to $6.00 \mathrm{~g} / \mathrm{L} \cdot \mathrm{h}$, with an average of $11.33 \mathrm{~g} / \mathrm{L}, 0.40 \mathrm{~g} / \mathrm{g}$, and $4.21 \mathrm{~g} / \mathrm{L} \cdot \mathrm{h}$, respectively (Fig. 2b). Comparing to glucose, the fermentation using BSG hydrolysate similarly presented the reduced fermentation time and the improved productivity of butyrate. Notably, the selectivity significantly increased $(6.77 \mathrm{~g} / \mathrm{g} v s .4 .06 \mathrm{~g} / \mathrm{g})$ in the fermentation of BSG hydrolysate.

Fig. 2 Kinetics (a) and long-term stability (b) of repeated-batch fermentation from BSG hydrolysate with C. tyrobutyricum immobilized in MBFBB. Results are expressed as the mean value and standard deviation of duplicate samples. (p-value ${ }^{*}<0.05 ; * \star<0.01 ; * \star *<0.001 ;$ )

\section{Fed-batch fermentation from BSG hydrolysate in MBFBB}

When the fed-batch fermentation was applied, the titer of butyrate steadily increased with the addition of concentrated BSG hydrolysate throughout the whole process (Fig. 3). The butyrate productivity (3.45 $\mathrm{g} / \mathrm{L} \cdot \mathrm{h}$ ) and glucose consumption rates were high from beginning to the 16th hour, and then decreased gradually. At the end of the fermentation ( $36 \mathrm{~h}$ ), the highest titer of $\sim 43.68 \mathrm{~g} / \mathrm{L}$ was obtained. The overall yield $(0.38 \mathrm{~g} / \mathrm{g})$ and productivity $(2.33 \mathrm{~g} / \mathrm{L} \cdot \mathrm{h})$ of butyrate were only $95.0 \%$ and $55.3 \%$ of those from the repeated-batch fermentation, which might be resulted from the increased product inhibition of high concentrations butyrate. However, the fed-batch fermentation using concentrated BSG hydrolysate exhibited a significantly higher selectivity $(11.67 \mathrm{~g} / \mathrm{g})$ for butyrate production than the batch fermentation $(6.77 \mathrm{~g} / \mathrm{g})$, which would definitely benefit to the product recovery and significantly reduce the total cost for biobutyrate production.

Fig. 3 Kinetics of fed-batch fermentation from BSG hydrolysate with $C$. tyrobutyricum immobilized in MBFBB. Results are expressed as the mean value and standard deviation of duplicate samples.

\section{Process design and techno-economic analysis for sodium butyrate production from BSG}

The preparation of sodium butyrate from BSG includes the alkali pretreatment, followed by the enzymatic hydrolysis of BSG, the fermentation of Clostridium tyrobutyricum in MFBB, and the downstream separation and production of sodium butyrate. Since the strong offensive odor is not suitable for animal feed industries application, sodium butyrate should be additionally encapsulated by gelatin and maltodextrin and then spray-dried to granules [20]. The flowchart for manufacturing encapsulated sodium butyrate from BSG is illustrated in Fig. 4. 
Fig. 4 A general process flowchart for manufacturing encapsulated sodium butyrate from BSG.

The techno-economic feasibility of sodium butyrate production from BSG was analyzed by SuperPro Designer. Based on $42.4 \%(\mathrm{w} / \mathrm{w})$ sugar yield from BSG ( 20\% cellulose, $20 \%$ hemicellulose) at \$29 per dry ton and $20 \mathrm{~g} / \mathrm{L}$ butyrate with a yield of $0.4 \mathrm{~g} / \mathrm{g}$ sugar and productivity of $4.21 \mathrm{~g} / \mathrm{L} \cdot \mathrm{h}$ in fermentation, butyrate can be produced at the cost of $\$ 1.5$ per kilogram for a 5,000-metric ton (MT) per year plant. The production cost would increase to $\sim \$ 2.49$ per kilogram if the plant size decreased to 1,000 MT. In contrast, the butyrate production at 5,000-MT scale would cost $\sim \$ 1.75$ per kilogram from barley and $\sim \$ 1.95$ per kilogram from glucose due to their much higher feedstock cost than BSG. The cost breakdowns for sodium butyrate production, including raw material costs (carbon source, nitrogen sources, enzyme, sulfuric acid, $\mathrm{NaOH}$, and solvent used in extraction), utility costs (nitrogen gas, electricity, steam, and water), equipment maintenance and depreciation costs, and labor costs are also shown in detail (Fig. 5). The raw materials accounted for $43 \%$ of the gross costs when glucose $(95 \%$ glucose, $\$ 450$ per MT) was used as a carbon source. Moreover, this ratio decreased to $37 \%$ for barley (65\% glucose, \$180 per MT) and 25\% for BSG (43\% glucose, \$29 per MT), respectively.

Fig. 5 Sodium butyrate production costs for 500, 1,000, 2,000, 3,000 and 5,000 MT plants using glucose, barley, and BSG, respectively, as feedstock in MBFBB fermentation processes. Cost data on capital equipment, energy, tax, interest, and labor were collected from existing butyrate fermentation plant and literature data.

The economics of sodium butyrate production could be influenced by the yield, productivity, and production scale of butyrate (Fig. 6). The estimated manufacturing costs for $30 \%(\mathrm{w} / \mathrm{v}$ ) sodium butyrate production from BSG was $\sim \$ 1500$ per MT with $0.4 \mathrm{~g} / \mathrm{g}$ yield, $0.421 \mathrm{~g} / \mathrm{L} \cdot \mathrm{h}$ productivity and $5,000 \mathrm{MT} / \mathrm{yr}$ scale. This value is much lower than the current cost of $\sim \$ 1,800 / \mathrm{MT}$ for the petroleum-derived butyrate. When the butyrate productivity is improved, the capital investment and product cost decrease, leading to an increased return of investment (ROI) [21]. At a sale price of $\$ 1.8$ per kilogram butyrate, $\mathrm{ROI}$ is $33.6 \%$ with a payback period of $\sim 3$ years for the 5,000-MT plant, whose estimated total capital investment is $\sim \$$ 14.6 MM. Compared with the butyrate cost from corn husk hydrolysate [20], butyrate production from BSG would be economically beneficial because of its higher ROI.

Fig. 6 Effects of annual production, butyrate yield and productivity on manufacturing cost of sodium butyrate from glucose, barley and BSG. (a) Effect of annual production on manufacturing cost. (b) Effect of butyrate yield on manufacturing cost. (c) Effect of butyrate productivity on manufacturing cost and return of investment.

\section{Discussion}

Mass transfer processes have a significant effect on microbial growth in industrial fermentations. Nutrients must be continuously replenished in the liquid layers closest to the microorganisms. Nutrients, such as glucose or ammonia of $1 \mathrm{~mol} / \mathrm{L}$ concentration in the fermentation might cause mass transfer issues [22]. In this study, the fermentation process with microbubbles coupling fibrous-bed bioreactor was 
superior to the traditional fibrous-bed bioreactor and had a shorter fermentation time between $12 \mathrm{~h}$ to $5 \mathrm{~h}$. It also had a two-fold higher butyric acid productivity from $2.24 \mathrm{~g} / \mathrm{L} \cdot \mathrm{h}$ to $4.36 \mathrm{~g} / \mathrm{L} \cdot \mathrm{h}$. The fermentable sugar utilization rate significantly increased by two-fold from 1.25 to $2.35 \mathrm{~g} / \mathrm{L} \cdot \mathrm{h}$. It can be concluded that microbubbles in MBFBB exhibit a positive effect on the fermentation and significantly improve the mass transfer efficiency between gas and liquid due to their small size, long existence time, and higher interface zeta potential.

The cellular ATPase activities and sugar utilization rate variations were investigated during the fermentation of butyric acid with MBFBB to evaluate the effects of microbubbles in MBFBB on the immobilized cells. It was observed that the immobilized cells in MBFBB had a significantly increased ATPase activity and sugar utilization rate, indicating that bacteria require a higher amount of ATP and glucose to maintain a higher growth rate. The metabolic pathway in C.tyrobutyricum is as follows: each unit of glucose can produce 3 units of ATP when producing butyric acid through the butyrate metabolic pathway, and each unit of glucose can produce 4 units of ATP when producing acetic acid through the acetic acid metabolic pathway. Therefore, in the rapid bacterial growth stage, cell metabolism requires more ATP, and the metabolic pathway is more inclined to the acetate metabolic pathway producing more ATP [2]. Our experimental results were consistent with these results, where the acetic acid concentration of the exponential phase of the fermentation increased faster than the end of the exponential phase. Furthermore, the decoupling effect of main products (butyrate and acetate) oxidative phosphorylation will interfere with establishing and maintaining the cell transmembrane $\mathrm{pH}$ gradient [23]. In order to maintain a proper intracellular and extracellular proton gradient, ATP must be consumed, and these protons should be pumped out of the cell by transmembrane ATPase. An active transmembrane ATPase is essential to prevent main products from acidifying the cytoplasm and maintaining the relative stability of intracellular $\mathrm{pH}$ [24]. In the fermentative production of butyric acid with MBFBB, the production rates of butyrate and acetate were much higher than the conventional FBB. A larger amount of ATP was required to maintain the relative stability of intracellular $\mathrm{pH}$.

Additionally, pure monosaccharides derived from crops are not sustainable and suitable substrates for the production of bulk biochemicals due to the high feedstock cost and impact on food supply. To overcome these limitations, the feedstocks for bio-butyrate production are gradually enlarging from food crops to inexpensive and renewable biomass, such as waste biomass [25]. Waste biomass as low-valued byproducts obtained from agriculture (wheat straw, oilseed rape straw, corn fiber, sugarcane bagasse, sorghum stalk, and corn husk) and forestry (waste paper) has been a focus of recent studies for butyric acid fermentation $[5,15-18,20,26]$. Unlike seasonal crop wastes, BSG can be obtained in large quantities at a low cost throughout the year. BSG mainly composed of the barley malt grain husks in mixture with part of the pericarp and seed coat layers of these grains, rich in sugar, protein, and minerals, is the main by-product of the beer brewing mill, and the annual production of BSG in China has been estimated at approximately 10 million MT per year [27]. As a biomass feedstock, BSG can not be directly utilized by Clostridium tyrobutyricum cells and must be pretreated and hydrolyzed to generate monosaccharides. Sulfuric acid combined with high temperature is one of the most commonly employed methods to hydrolyze biomass. However, the biomass acid pretreatment could generate various toxic by-products, 
including acids (coumaric, glucuronic, and formic acids), salts (neutralization products), 5-HMF, and furfural, which could exert inhibitory effects on the $C$. tyrobutyricum cells $[17,28]$. In order to avoid these inhibitors, alkali and enzymatic hydrolysis pretreatments of BSG were performed, and the highest sugar yield of $42.4 \%$ was achieved under the optimum hydrolysis conditions. The hydrolysates presented low toxicity $(0.001 \mathrm{mg} / \mathrm{mL} \mathrm{HMF}$ and $0.006 \mathrm{mg} / \mathrm{mL}$ furfural), and thus, it could eliminate the detoxification stage before fermentation. Moreover, a stable and reliable production of butyrate was obtained in repeated-batch and fed-batch fermentation processes using BSG hydrolyzate. The average butyrate titer, yield, productivity, and selectivity were $11.33 \mathrm{~g} / \mathrm{L}, 0.40 \mathrm{~g} / \mathrm{g}, 4.21 \mathrm{~g} / \mathrm{L} \cdot \mathrm{h}$, and $6.77 \mathrm{~g} / \mathrm{g}$, for repeated-batch fermentation and $43.68 \mathrm{~g} / \mathrm{L}, 0.38 \mathrm{~g} / \mathrm{g}, 2.33 \mathrm{~g} / \mathrm{L} \cdot \mathrm{h}$, and 11.67 ( $\mathrm{g}_{\text {butyrate }} / \mathrm{g}_{\text {acetate }}$ ) for fed-batch fermentation. The butyrate titer and yield obtained from the fermentation of BSG hydrolyzate were comparable to those of traditional monosaccharide substrates, such as glucose, xylose, and fructose. Moreover, the productivity and selectivity exceeded higher levels than other low-cost feedstocks.

Overall, the production cost of encapsulated sodium butyrate from petroleum-derived butyrate ( $\$ 1800$ per MT) for animal feed application is currently sold at US $\$ 2380-\$ 3174$ per MT. A similar product can be produced from BSG at a cost of US $\$ 1517-\$ 2100$ per MT, assuming a similar encapsulation process cost for the fermentation-derived sodium butyrate. With a large gross margin of more than $\$ 1000$ per MT, the valorization of BSG process is highly profitable and economically attractive.

In summary, the role of MBFBB in the fermentative production of butyrate by immobilized Clostridium tyrobutyricum cells from a renewable feedstock of BSG was evaluated in this study. Compared with the conventional FBB fermentation, two-fold shorter fermentation time and two-fold higher butyrate productivity were achieved. Furthermore, long-term stability and reliability for butyrate production were achieved and fed-batch fermentation using BSG hydrolysate produced a high butyrate titer with a significantly higher selectivity. The study results indicated that the MBFBB-based fermentation process with BSG is a robust and ecofriendly technique, which would provide insights into future development of commercial biobutyrate production derived from lignocellulosic waste biomass in beer factories.

\section{Methods}

\section{Cultures and medium}

Butyrate-producing strain Clostridium tyrobutyricum ZJUT1 was collected from strain Clostridium tyrobutyricum ZJU8235 provided by Professor Zhinan Xu from Zhejiang University. This strain was adapted in a fibrous bed bioreactor by the previously reported methods [29]. The fermentation culture medium contained (g/L): glucose 30; yeast extract 5; peptone 5; $\left(\mathrm{NH}_{4}\right)_{2} \mathrm{SO}_{4} 3 ; \mathrm{K}_{2} \mathrm{HPO}_{4} 1.5 ; \mathrm{MgSO}_{4} \cdot 7 \mathrm{H}_{2} \mathrm{O}$ $0.6 ; \mathrm{FeSO}_{4} \cdot 7 \mathrm{H}_{2} \mathrm{O}$ 0.03. The medium $\mathrm{pH}$ was adjusted to $\sim 6.5-7.0$ and sterilized at $121^{\circ} \mathrm{C}$ for $20 \mathrm{~min}$.

\section{Pretreatment and hydrolysis of brewer's spent grain}

Brewer's spent grain was provided by Hangzhou Cheerday Brewery Co. Ltd, Hangzhou, China. The BSG was dried in an oven at $45^{\circ} \mathrm{C}$ prior to the experimental runs. After alkaline pretreatment $(1 \mathrm{M} \mathrm{NaOH}$ 
solution for $40 \mathrm{~min}$ at $121^{\circ} \mathrm{C}$ ) of BSG, the pH of the solid fraction was controlled at 4.8 by adding $4 \mathrm{M}$ of hydrochloric acid. Glucose was extracted by enzymatic hydrolysis using cellulase $(12000 \mathrm{U} / \mathrm{mL}$, Hunan Hongying Biotech Co. Ltd) and $\beta$-glucosidase (110 U/g, Jiangsu Ruiyang Biotech Co. Ltd) for $24 \mathrm{~h}$ at 50 ${ }^{\circ} \mathrm{C}$ and $200 \mathrm{rpm}$. Three-level orthogonal tests were designed to evaluate the effects of BSG biomass (5, $10,15 \%, \mathrm{w} / \mathrm{v})$ and $\mathrm{NaOH}$ concentration $(1,2,3 \%, \mathrm{w} / \mathrm{v})$ in the alkaline pretreatment step, as well as the $\operatorname{BSG}$ biomass $(5,10,15 \%, w / v)$, enzyme loading $\left(45,67.5,90, U_{\text {cellulase }} / g_{\text {substrate, }}\right.$ and $45,67.5,90, U_{\beta-}$ glucosidase $\left./ g_{\text {substrate }}\right)$ in the enzymatic hydrolysis step for better glucose yield. A maximum glucose yield of

$42.4 \%\left(\mathrm{~g}_{\text {glucose }} / \mathrm{g}_{\mathrm{BSG}}\right)$ was achieved (Table $\mathrm{S} 1$ ) under the optimized hydrolysis conditions. Then, the hydrolysate sugar compositions were determined and the generated monosaccharides contained 50.2 $\mathrm{g} / \mathrm{L}$ glucose and $3.0 \mathrm{~g} / \mathrm{L}$ xylose. A small amount of xylose in the hydrolysate might have been produced by the acid neutralization process. The BSG hydrolysate was collected by vacuum filter and stored in the refrigerator for future use.

\section{Fermentation in microbubbles coupling fibrous-bed bioreactor}

The MBFBB was composed of a 5-L stirred-tank fermenter containing an $\mathrm{N}_{2}$ sparger as microbubbles generator (The bubble generator diameter was $6 \mathrm{~cm}$ and the thickness was $1.2 \mathrm{~cm}$, the pore size was 40 $\mu \mathrm{m}$, and the plate had 680 pores per $\mathrm{cm}^{2}$ ) and a recirculation loop connected a 0.5 -L fibrous-bed bioreactor (FBB). The FBB was made of a glass column packed with a spirally wound cotton towel (300 $\times$ $300 \mathrm{~mm} ; 5 \mathrm{~mm}$ in thickness; with $>95 \%$ porosity) overlaid with a stainless-steel mesh. The bioreactor system was operated at $37^{\circ} \mathrm{C}$, and the $\mathrm{pH}$ was controlled at 6.0 with $2 \mathrm{M} \mathrm{NaOH}$ through an auto-sensing and dosing system. Before use, the bioreactor containing the medium was sterilized by autoclaving at $121^{\circ} \mathrm{C}$ for $30 \mathrm{~min}$, and then flushed with $\mathrm{N}_{2}$ for $30 \mathrm{~min}$. The fermenter was inoculated with $100 \mathrm{~mL}$ cells cultured in serum bottles and allowed to reach a cell density of $6.0\left(\mathrm{OD}_{600}\right)$ to start the fermentation. Later, the fermentation broth was circulated to immobilize cells through the fibrous bed at a pumping rate of $30 \mathrm{~mL} / \mathrm{min}$. After $48 \mathrm{~h}$ of continuous circulation, most of the cells were immobilized, and the cell density in the broth became constant. The fermentation broth in the fermenter was then removed, replaced with fresh medium, and increased the medium circulation rate to $100 \mathrm{~mL} / \mathrm{min}$ to start the fermentation. The batch fermentations were carried out with glucose to evaluate the role of microbubbles in the fermentative production of butyric acid with MBFBB. When sugars were almost depleted, the fermentation broth in the fermenter was removed and replaced with a fresh medium. Repeated batch fermentation and fed-batch fermentation with BSG hydrolysate were then carried out to evaluate the kinetics and possible effects of hydrolysate inhibitors on long-term process performance. $\mathrm{N}_{2}$ was supplied to MBFBB at $1 \mathrm{VVM}(\mathrm{L} / \mathrm{L} \cdot \mathrm{min})$ throughout the fermentation process. Samples were taken at regular intervals for the analysis of biomass, substrate, and product concentrations.

\section{Characterization of microbubbles generated by $\mathrm{N}_{2}$ sparger}

Microbubbles generated by $\mathrm{N}_{2}$ sparger were characterized with the images captured by a digital camera (EOS M5, Canon) in an illuminating field created by a halogen lamp (500 W). The captured images were 
analyzed by image processing software Digimizer [30]. Microbubbles image and the microbubble size distribution are illustrated in Fig. S1. The microbubbles size distribution was relatively concentrated and uniform and the bubble diameter was mainly distributed in the range of $0.2-0.25 \mathrm{~mm}$ with an average diameter of $0.22 \mathrm{~mm}$.

\section{Cellular ATPase activity Assay}

Clostridium tyrobutyricum cells were collected after $1,3,5$, and $7 \mathrm{~h}$ in repeated batch fermentation with glucose as the carbon source using microbubbles coupling or non-microbubbles coupling fibrous-bed bioreactors. Clostridium tyrobutyricum cell extract was prepared, and the cellular ATPase activity assay was strictly performed following the instructions of the ATPase test $\mathrm{kit}\left(\mathrm{Ca}^{2+} / \mathrm{Mg}^{2+} \mathrm{ATPase}\right)$ [29]. The ATPase activity unit was defined as the amount of $\mathrm{Ca}^{2+} / \mathrm{Mg}^{2+}$-ATPase decomposing ATP to produce 1 $\mu \mathrm{mol}$ of inorganic phosphorus per gram of cell per hour is one enzyme activity unit.

\section{Cellular sugar utilization rate assay}

Cell pellets were harvested by centrifugation at $8000 \mathrm{rpm}$ and $4^{\circ} \mathrm{C}$ for $5 \mathrm{~min}$, then the pellets were washed twice with equal volume of distilled water and resuspended in $10 \mathrm{~g} / \mathrm{L}$ glucose solution. After incubation at $37^{\circ} \mathrm{C}$ for $6 \mathrm{~h}$, the suspension aliquots were discarded to determine the glucose concentration $[16,17]$. Cellular sugar utilization rate was expressed as follows: cellular sugar utilization rate $(\mathrm{g} / \mathrm{g} \cdot \mathrm{h})=\left(\mathrm{W}_{0} \mathrm{\square}\right.$ $\left.\mathrm{W}_{\mathrm{G}}\right) / \mathrm{W} / 6$, where $\mathrm{W}_{0}$ is the initial weight of glucose, $\mathrm{W}_{\mathrm{G}}$ is the final weight of glucose, and $\mathrm{W}$ is the weight of wet bacteria.

\section{Analytical methods}

During fermentation, the cell concentration was determined at $600 \mathrm{~nm}\left(\mathrm{OD}_{600}\right)$ by a spectrophotometer, and the glucose concentration was determined by SBA Biosensor Analyzer (Biology Institute of Shandong Academy of Science. Shandong, China). The concentrations of other sugars in the hydrolysate were determined by the previously reported methods $[16,17]$, where HPLC-ELSD was used with Bio-Rad Aminex HPX-87H column. The butyrate and acetate concentrations were determined by gas chromatography with flame ionization detector and Stabilwax column (Restek 10624, USA) $\left(30 \mathrm{~m} \times 0.32 \mathrm{~mm} \times 0.25 \mu \mathrm{m}, d_{f}=\right.$ 0.25).

\section{Techno-economic analysis}

The economic feasibility for producing encapsulated sodium butyrate from BSG was analyzed using SuperPro Designer.

\section{Declarations}

\section{Authors' Contributions}


Linqi Zhao: Conceptualization, Validation, Formal analysis, Investigation, Writing original draft. Gaoya Sun: Investigation. Jing Jiang: Conceptualization, Validation, Writing - review \& editing. Li Chen:

Resources, Writing - review \& editing. Jin Huang: Conceptualization, Validation, Writing - review \& editing, Supervision, Project administration.

\section{Acknowledgements}

The authors would like to thank Zefeng Guo (Hangzhou Cheerday Brewery Co. Ltd, Hangzhou, China) for kindly supplying the Brewer's spent grain used in this work. The research was supported by grants from the Natural Science Foundation of China (NSFC) (No.21406204), and the Zhejiang Provincial Natural Science Foundation (No.LQ12B06005), PR China.

\section{Competing Interests}

The authors declare that they have no known competing financial interests or personal relationships that could have appeared to influence the work reported in this paper.

\section{Availability of data and materials}

All data generated or analyzed during this study are included in this published article and its supplementary information files.

\section{Consent for publication}

Not applicable.

\section{Ethics approval and consent to participate}

Not applicable.

\section{Funding}

Funding sources have been addressed in "Acknowledgements."

\section{References}

1. Wang J, Lin M, Xu M, Yang S. Anaerobic fermentation for production of carboxylic acids as bulk chemicals from renewable biomass. Adv Biochem Eng Biotechnol. 2016; 156: 323-61.

2. Fu H, Hu J, Guo X, Feng J, Zhang Y, Wang J. High-selectivity butyric acid production from Saccharina japonica hydrolysate by Clostridium tyrobutyricum. Ind Eng Chem Res. 2020; 59: 17147-55.

3. Huang J, Tang W, Zhu S, Du M. Biosynthesis of butyric acid by Clostridium tyrobutyricum. Prep Biochem Biotech. 2018; 48: 427-34. 
4. Ji Hye J, Lee D, Kim J, Park J. Effect of initial glucose concentrations on carbon and energy balances in hydrogen-producing Clostridium tyrobutyricum JM1. J Microbiol Biotechn. 2009; 19: 291-8.

5. Wei D, Liu X, Yang S. Butyric acid production from sugarcane bagasse hydrolysate by Clostridium tyrobutyricum immobilized in a fibrous-bed bioreactor. Bioresour Techonol. 2013; 129: 553-60.

6. Bao T, Feng J, Jiang W, Fu H, Wang J, Yang S. Recent advances in n-butanol and butyrate production using engineered Clostridium tyrobutyricum. World J Microb Biot. 2020; 36:138.

7. Luo H, Yang R, Zhao Y, Wang Z, Liu Z, Huang M, Zeng Q. Recent advances and strategies in process and strain engineering for the production of butyric acid by microbial fermentation. Bioresour Technol. 2018; 253: 343-54.

8. Raghavendran V, Webb J, Cartron M, Green J. A microbubble-sparged yeast propagation-fermentation process for bioethanol production. Biotechnol Biofuels. 2020; 13: 104.

9. Weber J, Agblevor F. Microbubble fermentation of Trichoderma reesei for cellulase production. Process Biochem. 2005; 40: 669-76.

10. Inan K, Sal F, Rahman A, Putman R, Agblevor F, Miller C. Microbubble assisted polyhydroxybutyrate production in Escherichia coli. BMC Res Notes. 2016; 9: 338.

11. Kaster J, Michelsen D, Velander W. Increased oxygen transfer in a yeast fermentation using a microbubble dispersion. Appl Biochem Biotechnol. 1990; 24: 469-84.

12. Hensirisak P, Parasukulsatid P, Agblevor F, Cundiff J, Velander W. Scale-up of microbubble dispersion generator for aerobic fermentation. Appl Biochem Biotech. 2002; 101: 211-27.

13. Azevedo A, Etchepare R, Calgaroto S, Rubio J. Aqueous dispersions of nanobubbles: generation, properties and features. Miner Eng. 2016; 94: 29-37.

14. Bredwell MD, Worden RM. Mass-transfer properties of microbubbles. 1. experimental studies. Biotechnol Prog. 1998; 14: 31-8.

15. Zhu Y, Wu Z, Yang S. Butyric acid production from acid hydrolysate of corn fibre by Clostridium tyrobutyricum in a fibrous-bed bioreactor. Process Biochem. 2002; 38: 657-66.

16. Huang J, Dai H, Yan R, Wang P. Butyric acid production from recycled waste paper by immobilized Clostridium tyrobutyricum in a fibrous-bed bioreactor. J Chem Technol Biot. 2016; 91:1048-54.

17. Huang J, Zhu H, Tang W, Wang P, Yang S. Butyric acid production from oilseed rape straw by Clostridium tyrobutyricum immobilized in a fibrous bed bioreactor. Process Biochem. 2016; 51: 1930-4.

18. Baroi GN, Baumann I, Westermann P, Gavala HN. Butyric acid fermentation from pretreated and hydrolysed wheat straw by an adapted Clostridium tyrobutyricum strain. Microb Biotechnol. 2015; 8: 874- 
19. Mussatto S. Brewer's spent grain: a valuable feedstock for industrial applications. J Sci Food Agr. 2014; 94: 1264-75.

20. Xiao Z, Cheng C, Bao T, Liu L, Wang B, Tao W, Pei X, Yang S, Wang M. Production of butyric acid from acid hydrolysate of corn husk in fermentation by Clostridium tyrobutyricum: kinetics and process economic analysis. Biotechnol Biofuels. 2018; 11: 164.

21. Huang J, Du Y, Bao T, Lin M, Wang J, Yang S. Production of n-butanol from cassava bagasse hydrolysate by engineered Clostridium tyrobutyricum overexpressing adhE2: kinetics and cost analysis. Bioresour Technol. 2019; 292: 121969.

22. Zhang W, Li Z, Agblevor F. Microbubble fermentation of recombinant Pichia pastoris for human serum albumin production. Process Biochem. 2005; 40: 2073-2078.

23. Gutierrez N, Maddox L. Product inhibition in a nonmotile mutant of Clostridium acetobutylicum. Enzyme Microb Technol. 1992; 14: 101-5.

24. O'Sullivan E, Condon S. Relationship between acid tolerance, cytoplasmic pH, and ATP and H+ATPase levels in chemostat cultures of Lactococcus lactis. Appl Environ Microb. 1999; 65: 2287-93.

25. Jiang L, Fu H, Yang H, Xu W, Wang J, Yang S. Butyric acid: applications and recent advances in its bioproduction. Biotechnol Adv. 2018; 36: 2101-17.

26. Sjöblom M, Matsakas L, Christakopoulos P, Rova U. Production of butyric acid by Clostridium tyrobutyricum (ATCC25755) using sweet sorghum stalks and beet molasses. Ind Crop Prod. 2015; 74 : 535-44.

27. Plaza P, Gallego-Morales L, Peñuela-Vásquez M, Lucas S, García-Cubero M, Coca M. Biobutanol production from brewer's spent grain hydrolysates by Clostridium beijerinckii. Bioresour Technol. 2017; 244: 166-174.

28. Fu H, Yang S, Wang M, Wang J, Tang I. Butyric acid production from lignocellulosic biomass hydrolysates by engineered Clostridium tyrobutyricum overexpressing xylose catabolism genes for glucose and xylose co-utilization. Bioresour Technol. 2017; 234: 389-96.

29. Jiang L, Wang J, Liang S, Cai J, Xu Z, Cen P, Yang S. Enhanced butyric acid tolerance and bioproduction by Clostridium tyrobutyricum immobilized in a fibrous bed bioreactor. Biotechnol Bioeng. 2010; 108: 31-40.

30. Patel S, Majumder S, Das P, Ghosh P. Ozone microbubble-aided intensification of degradation of naproxen in a plant prototype. J Environ Chem Eng. 2019; 7: 103102. 


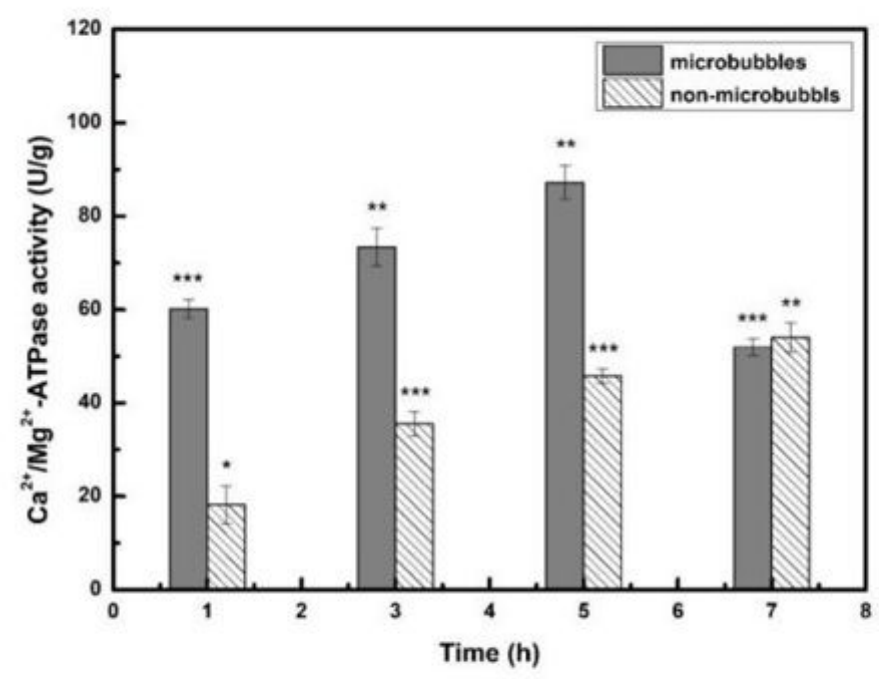

(a)

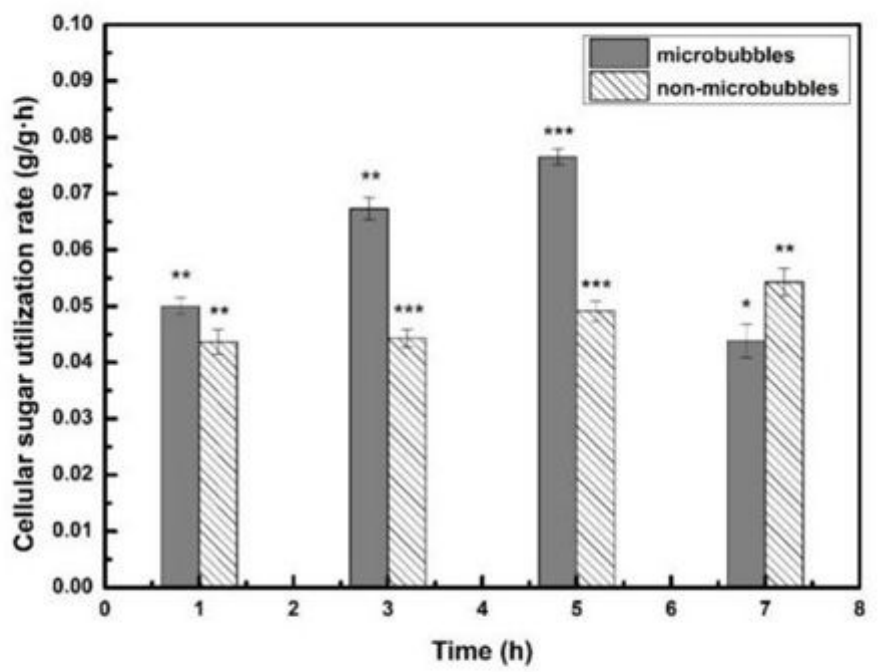

(b)

\section{Figure 1}

Effects of microbubbles on cellular ATPase activity (A) and sugar utilization rate (B) of Clostridium tyrobutyricum immobilized in MBFBB. Results are expressed as the mean value and standard deviation of duplicate samples. ( $p$-value $*<0.05 ; * \star<0.01 ; * \star *<0.001 ;$ )

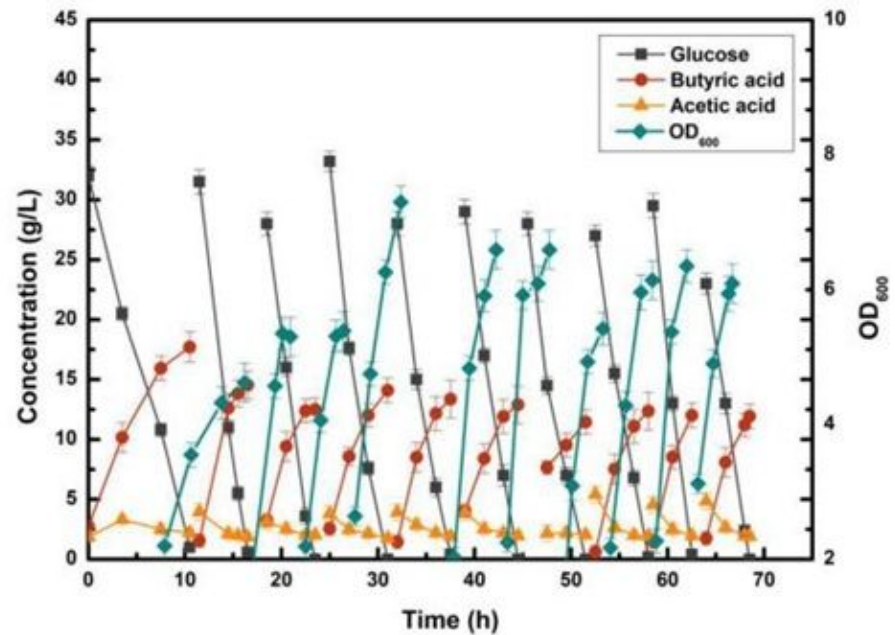

(a)

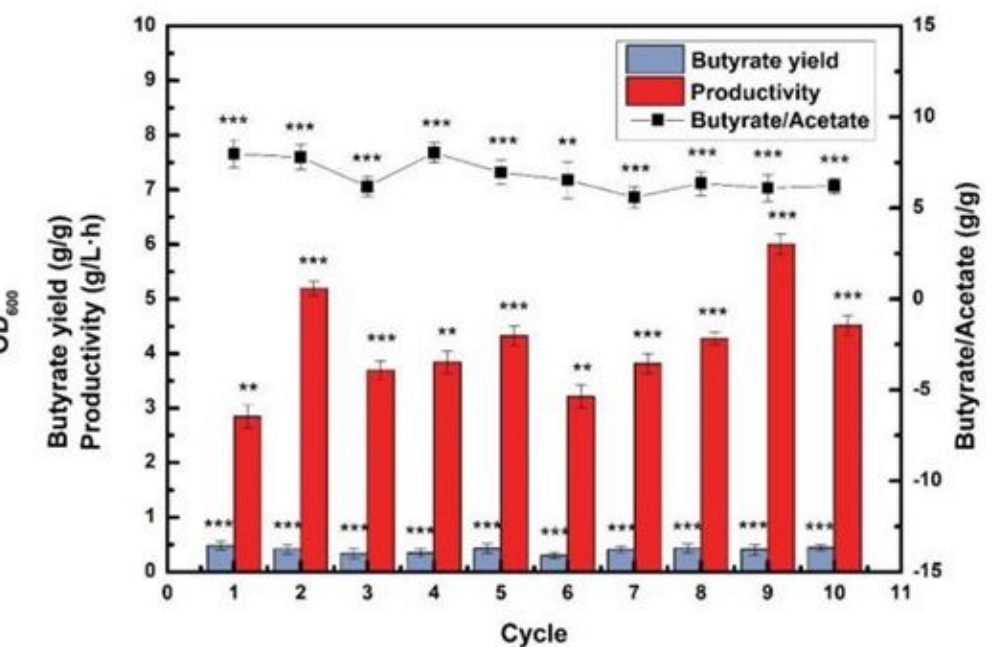

(b)

\section{Figure 2}

Kinetics (a) and long-term stability (b) of repeated-batch fermentation from BSG hydrolysate with C. tyrobutyricum immobilized in MBFBB. Results are expressed as the mean value and standard deviation 
of duplicate samples. (p-value *<0.05; $* *<0.01 ; * \star \star<0.001 ;)$

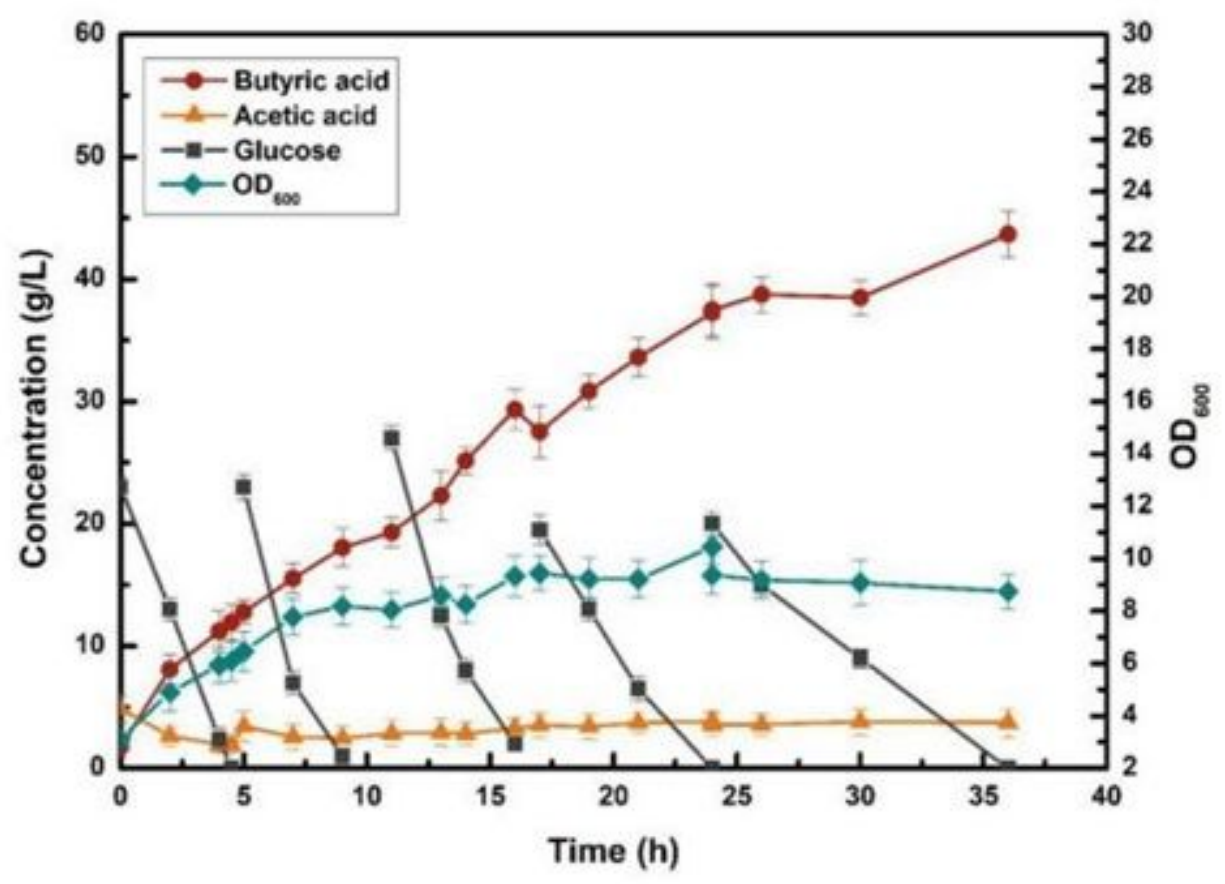

\section{Figure 3}

Kinetics of fed-batch fermentation from BSG hydrolysate with C. tyrobutyricum immobilized in MBFBB. Results are expressed as the mean value and standard deviation of duplicate samples. 


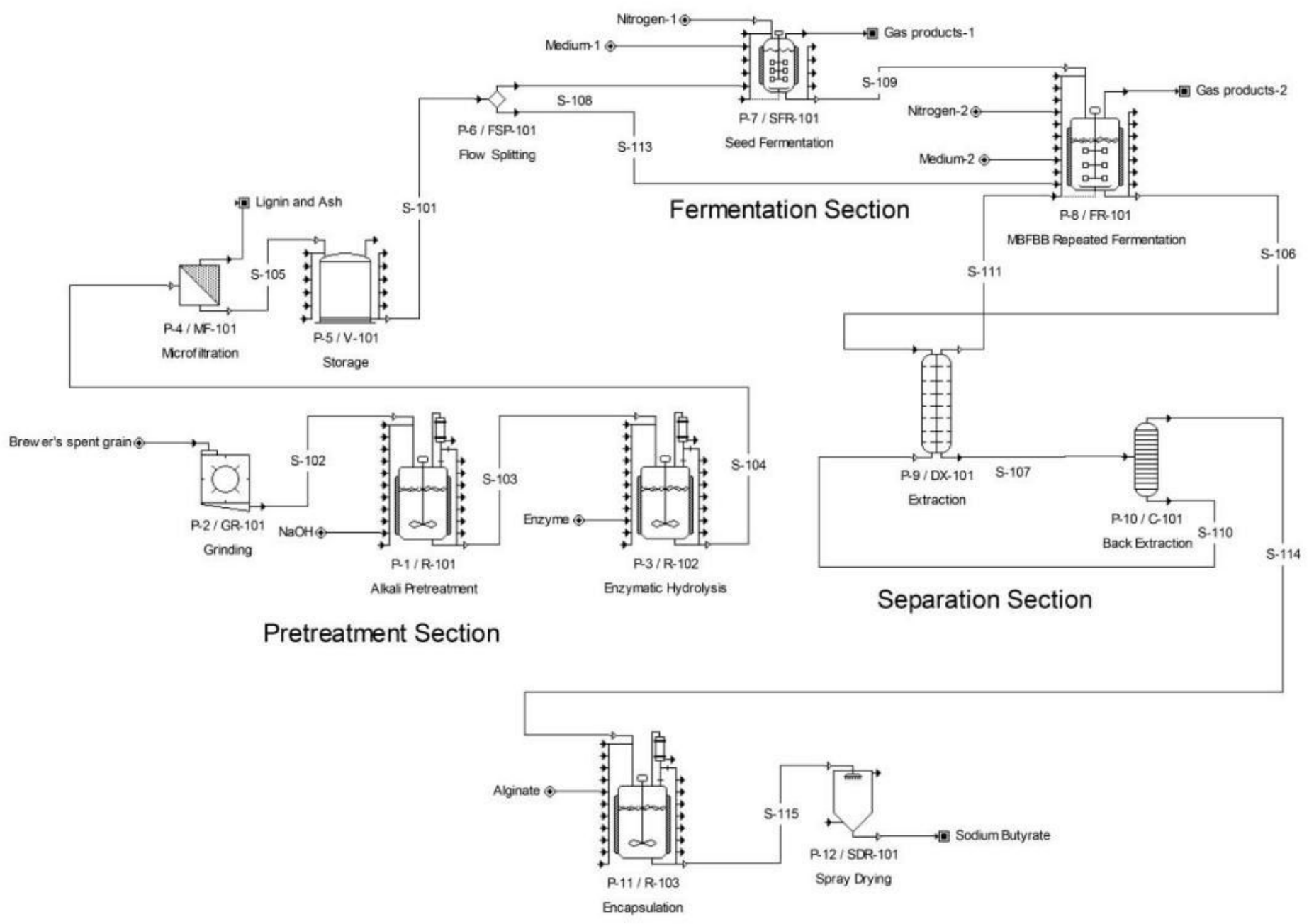

Figure 4

A general process flowchart for manufacturing encapsulated sodium butyrate from BSG. 

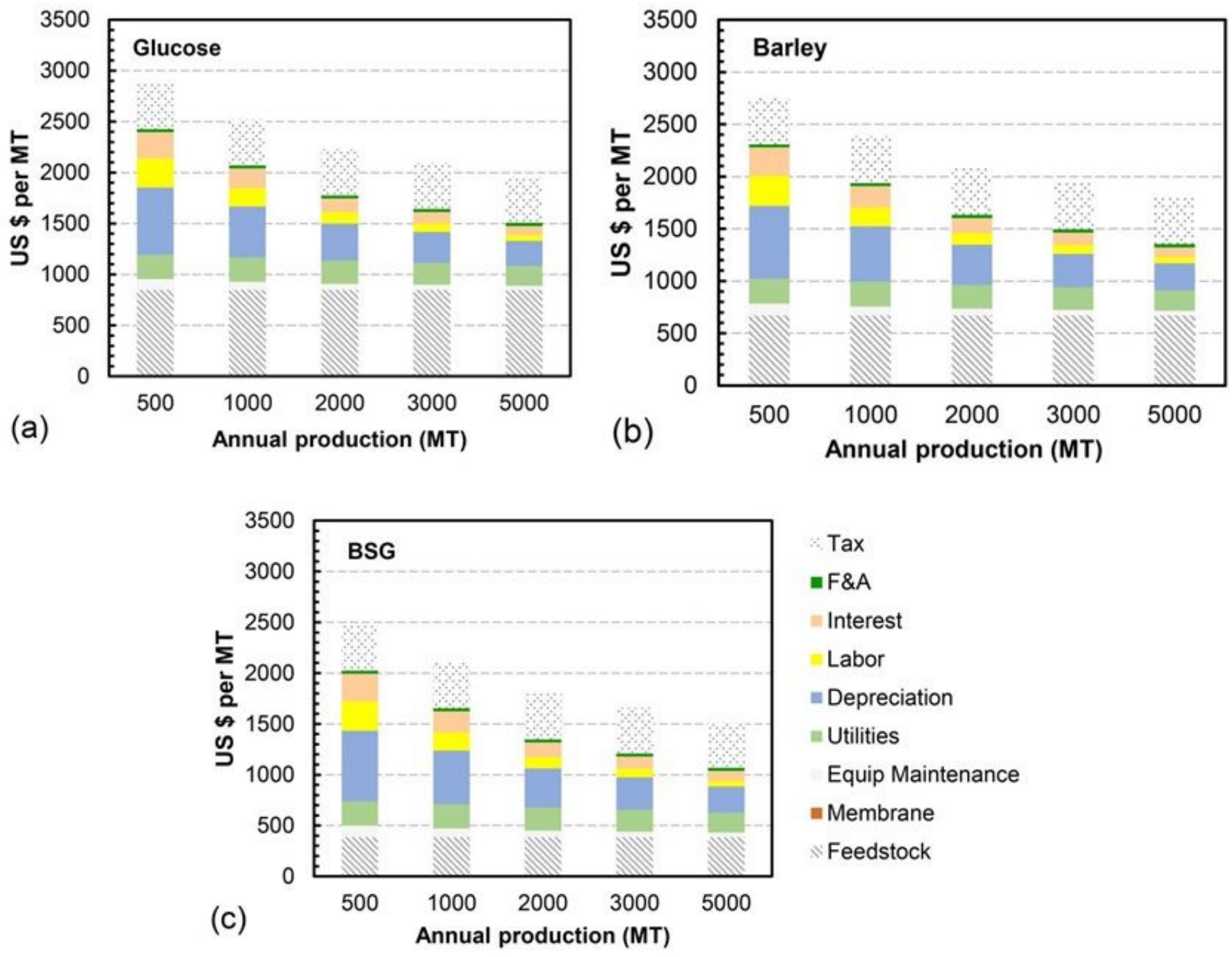

Figure 5

Sodium butyrate production costs for 500, 1,000, 2,000, 3,000 and 5,000 MT plants using glucose, barley, and BSG, respectively, as feedstock in MBFBB fermentation processes. 

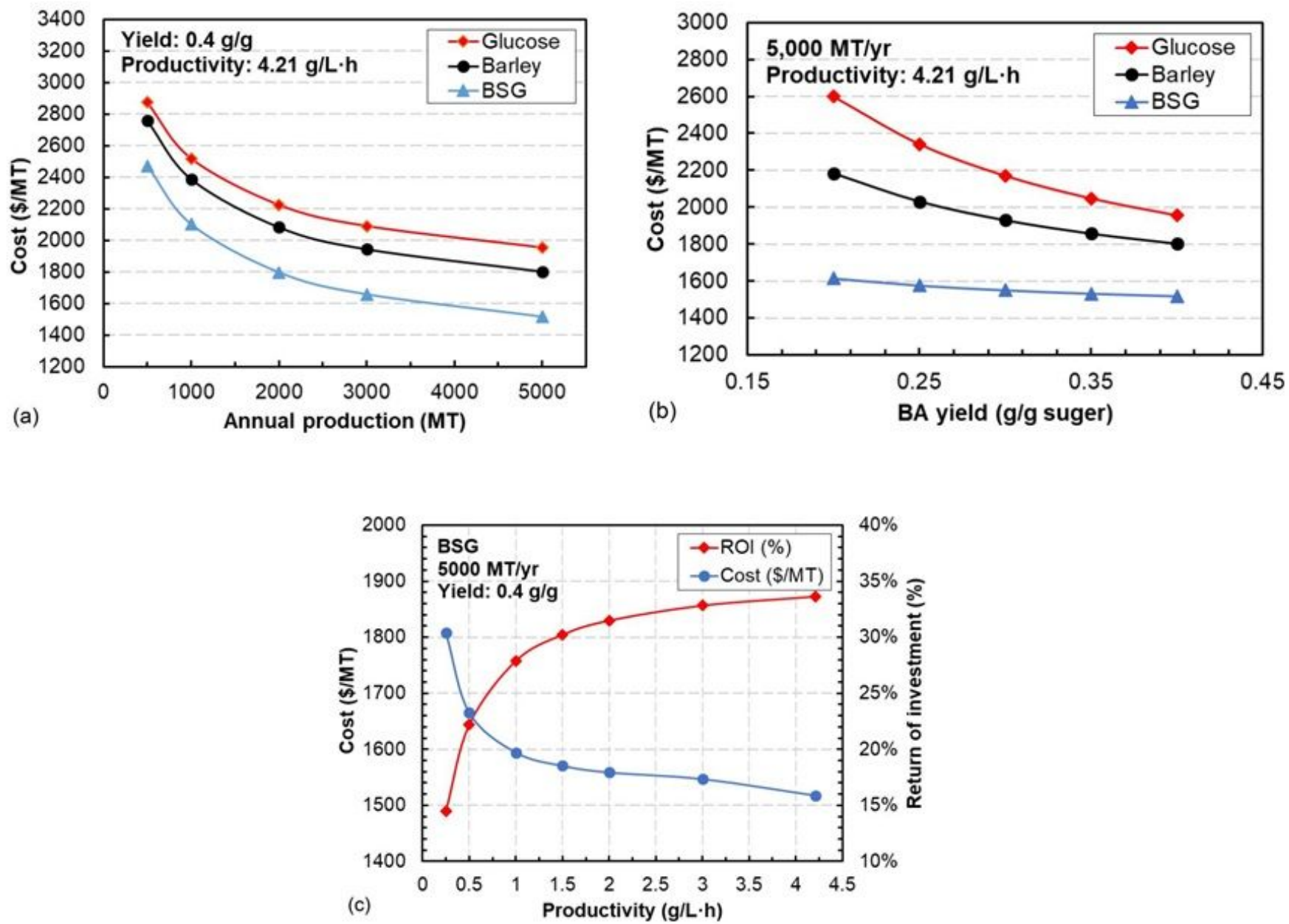

Figure 6

Effects of annual production, butyrate yield and productivity on manufacturing cost of sodium butyrate from glucose, barley and BSG. (a) Effect of annual production on manufacturing cost. (b) Effect of butyrate yield on manufacturing cost. (c) Effect of butyrate productivity on manufacturing cost and return of investment.

\section{Supplementary Files}

This is a list of supplementary files associated with this preprint. Click to download.

- Graphicfortableofcontents.pdf

- Supplymentary.doc 